\title{
Variables affecting long-term usage rate of sperm samples cryopreserved for fertility preservation in cancer patients
}

\author{
Stefania Ferrari ${ }^{1}$ | Alessio Paffoni ${ }^{2}$ (D) | Marco Reschini ${ }^{1}$ | Stefania Noli ${ }^{1,3}$ | \\ Chiara Dallagiovanna $^{1,3}$ | Cristina Guarneri ${ }^{1}$ | Francesca Filippi ${ }^{1}$ | Edgardo Somigliana ${ }^{1,3}$
}

\author{
${ }^{1}$ Obstetrics and Gynaecology Department, \\ Fondazione IRCCS Ca' Granda Ospedale \\ Maggiore Policlinico, Milan, Italy \\ ${ }^{2}$ Infertility Unit, ASST Lariana, Cantù, Come, \\ Italy \\ ${ }^{3}$ Department of Community and Clinical \\ Sciences, University of Milan, Milan, Italy \\ Correspondence \\ Alessio Paffoni, Infertility Unit, ASST \\ Lariana, Via Domea, 4 - 20036 - Cantù \\ (Come) - Italy. \\ Email: alessio.paffoni@alice.it
}

\begin{abstract}
Background: Previous evidence highlighted that only a minority of men who banked their semen before cancer therapies subsequently used their frozen samples. This may question the economical validity of sperm cryopreservation programmes. However, in most contributions, the duration of follow-up was insufficient to draw robust information on the real rate of use.
\end{abstract}

Objectives: To shed more light on the potential benefits of cryopreservation programmes.

Materials and methods: Men who cryopreserved their semen in a public hospital for a diagnosis of cancer between 1986 and 2009 were retrospectively reviewed. The rate of use as well as the possible determinants was investigated.

Results: The median time of follow-up was 12 [IQR: 7-16] years. One hundred fortyfour patients out of $1,524(9.4 \%, 95 \% \mathrm{Cl}$ : $8.1 \%-11.0 \%)$ used their frozen samples of whom $64 \%$ were azoospermic. The rate of men achieving parenthood with frozen semen was $46 \%$. Predictive factors of use were older age at the time of storage, lower sperm count at the time of storage and a diagnosis of testicular cancer. The impact of this latter factor was also supported by the lower frequency of azoospermia after cancer treatment in these patients.

Discussion: Cost-beneficial studies are warranted to assess and possibly improve the economical validity of sperm banking.

Conclusion: The usage rate of frozen sperm in cancer patient is low, even extending the duration of follow-up.

KEYWORDS

cancer, effectiveness, fertility preservation, semen, spermatozoa

\section{1 | INTRODUCTION}

In the last decades, therapeutic advances have improved the longterm survival of cancer patients. However, cancer treatments can cause side effects, including impairment of a boy's or man's fertility. ${ }^{1}$
Changes to fertility may be temporary or permanent according to several factors, but it has to be recognized that it is often impossible to accurately predict a priori the fertility status of a specific subject after oncological therapies. ${ }^{1}$ This is the reason why many highlevel guidelines, including the American Society for Reproductive 
Medicine (ASRM), ${ }^{2}$ the American Society of Clinical Oncology $(\mathrm{ASCO})^{3}$ and the National Institute for Health and Care Excellence $(\mathrm{NICE})^{4}$ strongly suggest to consider fertility preservation prior to initiate cancer treatments. In a recent systematic review of our group that included a large number of patients (11 798), we reported that only $8 \%$ ( $95 \%$ confidence interval- $\mathrm{Cl}$ : $8 \%-9 \%$ ) actually used their frozen semen, of whom $49 \%$ (95\% Cl: 44\%-53\%) achieved parenthood. ${ }^{5}$ Evidence from more recent contributions not included in our systematic review did not provide more comforting evidence, with reported rates of use varying between $3 \%$ and $11 \% .{ }^{6-8}$ However, these crude proportions may be an underestimation of the real figure. The rate of use is expected to increase by extending the duration of follow-up. Accordingly, in our review, when we plotted the rate of use with the median/mean length of follow-up of the included series, a positive correlation emerged $\left(R^{2}=.45\right) .{ }^{5}$ In addition, in these studies, only a minority of patients ( $16 \%, 95 \% \mathrm{Cl}: 15 \%-17 \%)$ discarded their samples indicating that the majority of subjects did not make a definitive decision about the possibility of using their frozen sperm in the future. ${ }^{5}$ This evidence is however indirect and, overall, there is the need to provide more reliable information on the rate of use in the long term.

The present study was designed to address this issue. Specifically, we reviewed our long-term experience of sperm banking for cancer patients in order to provide an estimate of the long-term usage rate of frozen sample and to identify a priori predictive factors for future use.

\section{2 | MATERIALS AND METHODS}

All male cancer patients who cryopreserved semen in the biobank of the Assisted Reproductive Technology (ART) Unit of the Fondazione IRCCS Cà Granda Ospedale Maggiore Policlinico in Milan were considered. In our Unit a sperm banking programme has been available since $1986 .^{9}$ The analyses included patients who cryopreserved semen until 2009 to have a follow-up period deemed to be significant. The study was approved by the local Ethics Committee (Comitato Etico Area B, Milan). All patients involved in the study provided a prospective written consent for their data to be used for research purposes, however, a specific informed consent was waived by the Ethics Committee based on the retrospective design of the study. The work has been carried out in accordance with the Code of Ethics of the World Medical Association (Declaration of Helsinki) and Uniform Requirements for manuscripts submitted to biomedical journals.

We used our database to collect basal characteristics of patients (including age at semen preservation, semen variables at the time of preservation, oncological diagnosis, previous children), main outcome of the study (use of frozen samples and time to use) and secondary outcomes (duration of follow-up, disposal of samples, post-treatment semen analysis). Inconsistencies were solved by consulting personal charts. Details of our programme are reported elsewhere. ${ }^{9,10}$ Of relevance here is that costs of the maintenance in the bank have obviously slightly changed over time but never consistently. Since 1999, the cost for biobanking is euro 100 (or its corresponding value in the previous currency) per year without additional costs for the procedure of freezing and it has never changed. As explained at the moment of cryopreservation, a post-oncological treatment sperm analysis (at least 12 months after the end of treatment) was advised and offered to patients at our centre. In case of semen analyses performed in other laboratories, patients were asked to send their lab reports. This information was used to assess the rate of azoospermia: however, for the specific evaluation of semen variables presented in this study, we decided to exclude cases exclusively performing semen analyses in external laboratories to improve homogeneity. An active follow-up was performed with phone calls in 2013-2014. This consented us to obtain adjourned information, to increase the referral for post-treatment semen analysis, to obtain more disposal and to fill missed data that were absent in our charts or in the database. The presented analyses were performed in 2015 using these available and adjourned data. The delay in submission and publication of the present study was due to organizational internal problems.

Statistical analyses were performed using Statistical Package for Social Science (SPSS) for Windows, Version 18.0 (SPSS Inc). The variable 'Cancer diagnosis' was simplified into three main categories: testicular cancer, haematological cancer and other type of cancer. Reference values of semen analyses were based on WHO parameters. ${ }^{11}$ Albeit available, data on semen morphology was not reported because of the drastic changes in the methodology for assessments occurring over the years (Cooper et al. 2010) ${ }^{11}$. Statistically significant differences were determined using Fisher's exact test, chisquared test, Student's t test or Kruskal-Wallis test, as appropriate. The Kaplan-Meier estimate was used to compute the cumulative rate of frozen sperm use over time. Time of follow-up was censored at the moment of sperm usage or corresponded to the time of follow-up (2015, when analyses were done). A Cox regression analysis was performed to determine the influence on the usage rate of the following variables: age at cryopreservation, cancer type, total sperm number per ejaculate at the time of cryopreservation and number of children before therapy. In addition, we performed a binomial logistic regression aimed at ascertain the effects of independent variables (the same reported for Cox regression plus time between cryopreservation and follow-up) on the likelihood that patients use their frozen sperm samples or show azoospermia at post-treatment sperm analysis. A binomial exact distribution model was used to estimate the $95 \% \mathrm{Cl}$ of proportions.

\section{3 | RESULTS}

\subsection{Population and semen analysis at cryopreservation}

Out of 1682 referred cancer patients between 1986 and 2009, 1524 (91\%) produced at least one sperm sample suitable for 
cryopreservation and were included in the study. Median age at the time of sperm banking was 29 years (interquartile range, IQR: 25-34) (range, 14-66 years). A median of 2 (range 1-5) ejaculates obtained in 2-5 days per patient were cryostored. Cancer diagnoses were as follows: testicular cancer, $n=660$ (43\%); Hodgkin Lymphoma (HL), $\mathrm{n}=443$ (29\%); non-Hodgkin lymphoma (NHL), $\mathrm{n}=134$ (9\%); leukaemia, $n=74$ (5\%); other solid tumours, $n=213$ (14\%). Sperm variables significantly differed according to cancer diagnosis. Median $[\mathrm{IQR}]$ semen volume in testicular, haematological and others was 3.0 [2.0-4.0], 2.7 [1.8-3.8] and 2.8 [2.0-4.0] mL, respectively $(P=.003)$. Median [IQR] sperm concentration was 29 [11-59], 56 [24-100] and 46 [18-82] million/ml, respectively $(P=.001)$. Median [IQR] of total sperm number was 81 [30-170], 128 [58-280] and 116 [42-238] million, respectively $(P=.001)$. Median [IQR] progressive motility was 45\% [34\%-55\%], 40\% [30\%-52\%] and 40\% [30\%-50\%], respectively $(P=.002)$. Semen characteristics at the time of storage according to diagnosis and WHO reference values are reported in Table 1.

\subsection{Usage rate}

The median time from storage to use or to the last follow-up for the entire cohort was 12 [IQR: 7-16] years (range 0-28 years). One hundred forty-four patients $(9.4 \%, 95 \% \mathrm{Cl}: 8.1 \%-11.0 \%)$ used their cryopreserved sperm samples. Nearly half of them performed the ART procedure in our Centre ( $n=68,47 \%), 62$ (43\%) transferred their samples to other clinics and the remaining 14 (10\%) used their samples both in our and other units. Among the 95 patients with available follow-up data on the outcome of ART with their frozen sperm, 50 obtained a pregnancy (53\%, 95\% Cl: 42\%-63\%). Live births were documented in 39 out of the 45 pregnant women with complete follow-up (87\%, 95\% Cl: 73\%-94\%). Among patients who reported a semen analyses at the time of use of the sperm sample ( $n=75)$, the rate of azoospermia was $61 \%(95 \% \mathrm{Cl}: 49 \%-72 \%)$. In nonazoospermic patients, median [IQR] total sperm number was 9.2 [0.3-20] million with $77 \%$ of subjects showing a total sperm number below the 5 th percentile of WHO reference values.
The Cox regression analysis identified three factors that were significantly associated with the use of frozen semen: (a) age (years) at the time of cryostorage $(\exp (B)=1.056,95 \% \mathrm{Cl}$ : 1.033-1.081, $P=.001$ ), (b) cancer diagnosis (haematological versus testicular tumours $\exp (B)=1.723,95 \% \mathrm{Cl}: 1.176-2.525, P=.005$; other diagnoses versus testicular $\exp (B)=1.974,95 \% \mathrm{Cl}: 1.167-3.338, P=.011)$ and (c) number of spermatozoa/ejaculate (in millions) $\exp (B)=0.999$, $95 \% \mathrm{Cl}$ : 0.998-1.000, $P=.029)$. The logistic regression analysis confirmed these results with a significant contribution to the model of the three variables. However, the model explained only $3.7 \%$ of the variance in usage rate and correctly classified $91 \%$ of cases. The area under the ROC curve was $0.629(95 \% \mathrm{Cl}$ : 0.582-0.676) which is a poor level of discrimination according to Hosmer et $\mathrm{al}^{12}$

Cumulative use rates of frozen semen are shown in Figure 1. The cumulative rate of use at 10 and 20 years was $8.6 \%$ and $12.0 \%$, respectively. A log rank test was run to determine if there were differences in the survival distribution for the different types of cancer diagnosis. A significant difference $(P<.01)$ emerged in the survival distribution between testicular tumours (cumulative use rate of frozen semen at 10 and 20 years was $5.8 \%$ and $9.4 \%$, respectively) compared to haematological (10.1\% and $14.2 \%$ ) or other malignancies (12.6\% and $12.6 \%$ ).

Globally, the median time between sperm banking and use of cryopreserved spermatozoa was 5.0 [IQR: 4.0-6.0] years (range 0-18 years). According to the diagnosis, the median [IQR] time to use of cryopreserved spermatozoa was 6.0 [5.2-6.8], 6.0 [4.8-7.2] and 3.0 [2.2-3.8] years for testicular, haematological and other types of cancer, respectively $(P=.02)$.

\section{3 | Post-treatment sperm analysis}

A total of 518 patients (34\%) performed a post-treatment sperm analysis. Sperm analysis in azoospermic men was performed after a median time of 48 [IQR: 31-46] months from cryopreservation and confirmed after 2 or 3 weeks. Azoospermia was documented in 106 subjects (20\%, 95\% Cl: $17 \%-24 \%)$. According to cancer diagnosis, the rate of azoospermia was $12 / 231$ (5\%, 95\% Cl: 3\%-9\%),

TABLE 1 Semen characteristics at the time of storage according to diagnosis

\begin{tabular}{|c|c|c|c|c|c|c|c|}
\hline \multirow[b]{2}{*}{ Cancer Diagnosis } & \multirow[b]{2}{*}{$\mathrm{N}(\%)$} & \multicolumn{3}{|c|}{ Total sperm number ${ }^{*}$} & \multicolumn{3}{|c|}{ Total progressive sperm number } \\
\hline & & $\leq 5$ th centile & 5th-50th centile & $\begin{array}{l}>50 \text { th } \\
\text { centile }\end{array}$ & $\leq 5$ th centile & $\begin{array}{l}\text { 5th-50th } \\
\text { centile }\end{array}$ & $\begin{array}{l}>50 \text { th } \\
\text { centile }\end{array}$ \\
\hline Testicular & $660(43 \%)$ & $202(31 \%)^{a}$ & $380(57 \%)$ & $78(12 \%)^{a, b}$ & $186(28 \%)^{a}$ & $410(62 \%)$ & $64(10 \%)^{a, b}$ \\
\hline Haematological & $651(43 \%)$ & $120(18 \%)^{a, b}$ & $356(55 \%)$ & $175(27 \%)^{a}$ & $133(20 \%)^{a}$ & $383(59 \%)$ & $135(21 \%)^{a}$ \\
\hline Other & 213 (14\%) & $53(25 \%)^{b}$ & $110(52 \%)$ & $50(23 \%)^{b}$ & $54(25 \%)$ & $125(59 \%)$ & $34(16 \%)^{b}$ \\
\hline Total & 1.524 & 375 (25\%) & $846(55 \%)$ & 303 (20\%) & $373(25 \%)$ & 918 (60\%) & 233 (15\%) \\
\hline
\end{tabular}

Note: Data are reported as number (\%). Sperm characteristics refer to the best available ejaculate per patient.

Values with same superscripts within columns are significantly different $(P<.05)$.

*Based on WHO reference intervals for fertile men (Cooper et al, 2009): 5 th centile $=39$ millions, 50 th centile $=255$ millions.

${ }^{* *}$ Based on WHO reference intervals for fertile men (Cooper et al, 2009): 5 th centile $=12.5$ millions, 50 th centile $=140$ millions. 

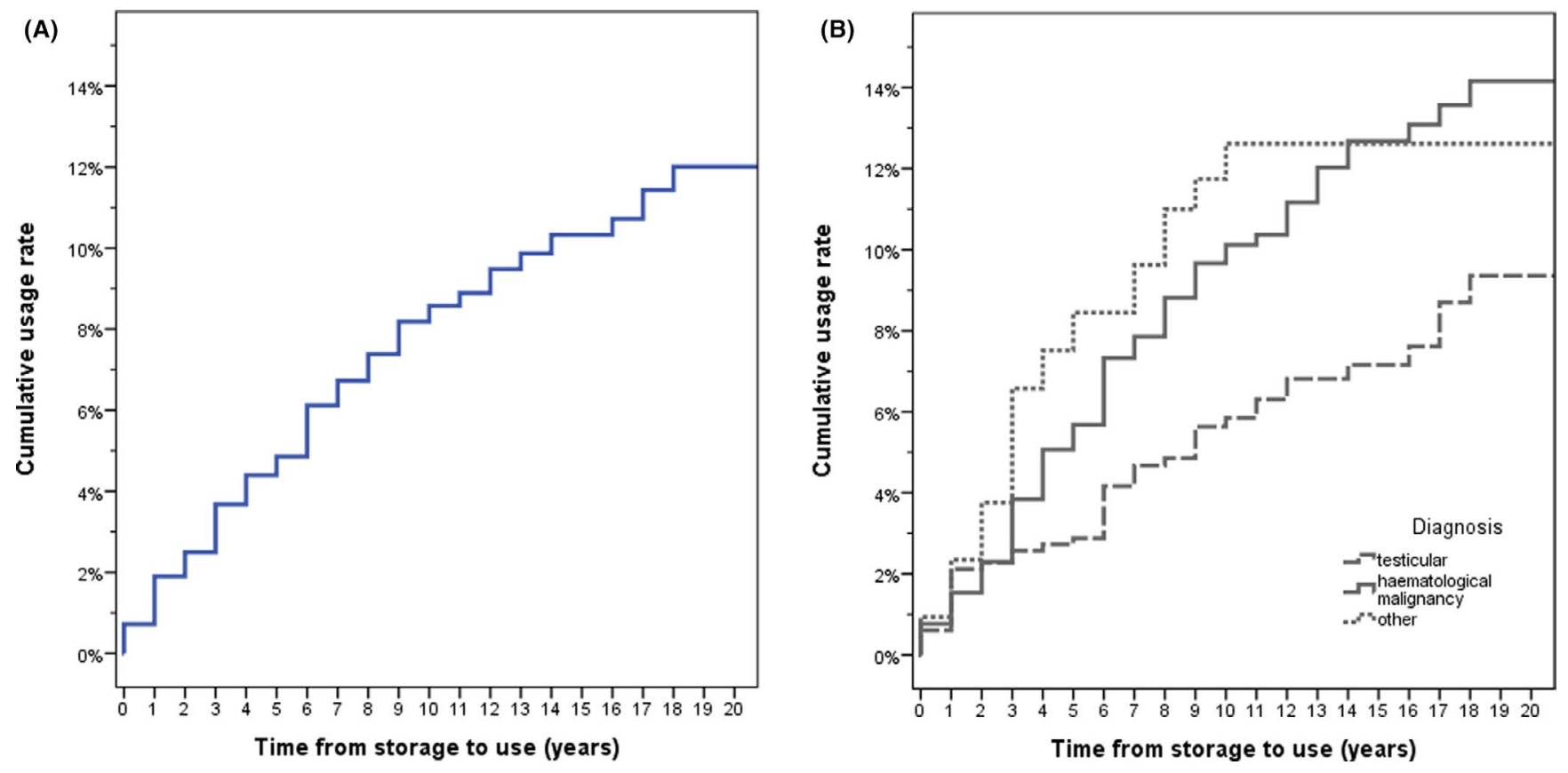

FIGURE 1 Cumulative rate of use of frozen semen for the whole cohort (A) and according to the diagnosis (B). The graph was plotted using Kaplan-Meier estimates. The curve was truncated at 20 years when data were available for $85 \%$ of the cohort

TABLE 2 Sperm characteristics at post-therapy control according to cancer diagnosis

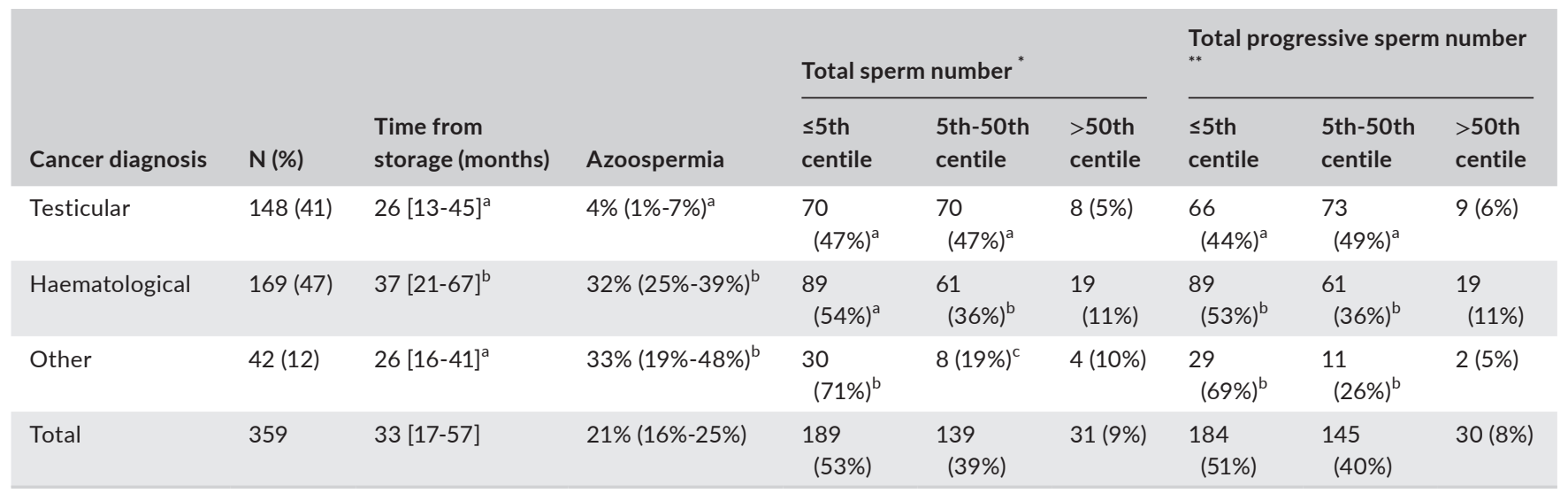

Note: Data are reported as number (\%), percentage (95\% confidence interval) or median and interquartile range [IQR].

Values with different superscripts within columns are significantly different $(P<.05)$.

*Based on WHO reference intervals for fertile men (Cooper et al, 2009): 5 th centile $=39$ millions, 50 th centile $=255$ millions.

**Based on WHO reference intervals for fertile men (Cooper et al, 2009): 5th centile $=12.5$ millions, 50th centile $=140$ millions.

76/234 (32\%, 95\% Cl: 27\%-39\%) and 18/53 (34\%, 95\% Cl: 23\%-47\%) in testicular, haematological and other cancer types, respectively $(P=.001)$. A binomial regression was performed to ascertain the effects of age at cryopreservation, total sperm count, diagnosis and time from storage to post-treatment sperm analysis on the likelihood that patients showed azoospermia. The model explained $21.4 \%$ of the variance in azoospermia and correctly classified $79 \%$ of cases. Specificity was $98 \%$, but sensitivity was as low as $9 \%$. Two predictor variables were found to be statistically significant: (a) time from cryopreservation to post-treatment sperm analysis $\exp (B)=1.096$, 95\% Cl: 1.033-1.163, $P=.003$ ), (b) cancer diagnosis (haematological versus testicular tumours $\exp (B)=7.614(95 \% \mathrm{Cl}$ : 3.896-14.881, $P<.001$; other diagnoses versus testicular $\exp (B)=9.320(95 \% \mathrm{Cl}$ : 4.002-21.708, $P=.005)$. In the subgroup of patients showing azoospermia at post-treatment analysis, the rate of users was similar among different cancer diagnoses: $42 \%, 45 \%$ and $39 \%$ for testicular, haematological and other cancers, respectively. Table 2 reports detailed information on the sperm variables for the subset of patients ( $n=351$ ) who performed semen analyses in our Unit. The percentage of patients showing a total/progressive sperm count below the 5 th centile compared to the reference fertile population significantly increased in the three groups of cancer diagnosis compared to basal 
TAB LE 3 Sperm characteristics before and after therapy according to cancer diagnosis

\begin{tabular}{|c|c|c|c|c|c|c|c|}
\hline Cancer diagnosis & $\mathrm{N}$ & \multicolumn{3}{|c|}{ Total sperm number (million) } & \multicolumn{3}{|c|}{ Total progressive sperm number (Million) } \\
\hline Haematological & 169 & $155[80-307]$ & $26[0-150]$ & 0.001 & $63[25-132]$ & $8[0-6]$ & 0.001 \\
\hline Other & 42 & 99 [49-200] & $9[0-50]$ & 0.001 & 37 [7-91] & $3[0-20]$ & 0.001 \\
\hline
\end{tabular}

TAB LE 4 Reasons for frozen sperm disposal according to cancer diagnosis

\begin{tabular}{|c|c|c|c|c|c|c|}
\hline Cancer Diagnosis & $\mathrm{N}$ & \multicolumn{5}{|c|}{ Reasons for the elimination of samples } \\
\hline Testicular & 288 & $5(2 \%)^{a}$ & $94(33 \%)^{a}$ & $26(9 \%)$ & $13(5 \%)$ & $150(52 \%)^{\mathrm{a}}$ \\
\hline Haematological & 274 & $58(21 \%)^{b}$ & $48(18 \%)^{b}$ & $14(5 \%)$ & $17(6 \%)$ & $137(50 \%)^{\mathrm{a}}$ \\
\hline Other & 86 & $35(41 \%)^{c}$ & $11(13 \%)^{b}$ & $4(5 \%)$ & $5(6 \%)$ & $31(36 \%)^{b}$ \\
\hline
\end{tabular}

Note: Data are reported as number (\%).

Values with different superscripts within columns are significantly different $(P<.05)$.

values $(P<.01)$. With regard to the same group of patients, a comparison between pre and post-treatment is reported in Table 3. A significant reduction in the number of total and progressive sperm cells per ejaculate was recorded in all diagnostic groups.

\section{4 | Disposal}

Out of 1380 patients who did not use their frozen spermatozoa, 648 (47\%) asked to discard their samples. A specific reason for sperm disposal was reported in about half of this sample $(n=330)$. The percentage of patients who asked to discard their unused frozen sperm samples was similar among the three types of cancer diagnosis: $47 \%, 48 \%$ and $46 \%$ for testicular, haematological and other types of cancers, respectively $(P=.83)$. However, the reason for sperm disposal varied according to cancer diagnosis, as shown in Table 4. The rate of patients asking to discard their sample was nearly double in non-azoospermic patients compared to azoospermic patients at post-treatment analysis ( $\mathrm{OR}=2.02,95 \% \mathrm{Cl}: 1.20-3.40)$.

\section{4 | DISCUSSION}

Our previous systematic review on the use and reproductive outcomes of sperm cryopreservation for cancer highlighted that the overall effectiveness of these programmes may be lower than previously thought. Indeed, only one in ten subjects thawed their samples and not all achieved parenthood. ${ }^{5}$ However, most studies included in this review had important pitfalls, such as, in particular, the lack of information on the cumulative usage rate in the long term. This point is crucial to figure out the real effectiveness of sperm cryopreservation programmes, considering in particular that parenthood is delayed until the late 30 s in the new generations. ${ }^{13}$ Our present study was actually designed to overcome the limitations of previous contributions. Nonetheless, we also highlighted a low rate of use (only 9.4\%). To note, the large sample size allowed us to shrink the $95 \% \mathrm{Cl}$ of this estimate (8.1\%-11.0\%) and the long-term follow-up (median of 12 years) consented us to infer reliable information up to 20 years of follow-up (with a usage rate increasing to $12.0 \%$ ). This latter evidence actually suggests that the cumulative rate of use may be only marginally increased by extending the duration of follow-up above 10 years. The modest impact of extending the length of follow-up is confirmed by the observed relatively short time between freezing and thawing in our analysis (median time of 5 years, IQR: 4-6 years). Noteworthy, our study also highlighted that the usage rate to estimate the potential benefits of sperm banking programmes in favouring fatherhood needs to be carefully interpreted. Indeed, it is only one of the aspects to be considered in terms of effectiveness and two additional aspects deserve consideration. First, a consistent proportion of men used their frozen semen despite not being azoospermic (only 61\% of users were actually azoospermic) and one cannot thus exclude that they could have achieved parenthood also with their fresh semen. Further studies are needed in order to shed more lights on the reasons why many non-azoospermic men decide to use frozen sperm samples; several aspects may contribute to this evidence such as impairment of sexual abilities, ejaculation disorders, severe impairment of post-treatment sperm quality or the diffuse-albeit unproven-idea that chemotherapy may determine some irreversible DNA damage that may increase the risk of congenital problems to the newborns. ${ }^{14,15}$

Second, $46 \%$ of men who thawed their semen achieved parenthood (calculated as $0.53 \times 0.87$, ie cumulative pregnancy rate per chance of reaching live births). This rate is in line with previous evidence (our systematic review depicted a $49 \%$ rate) and is 
somehow surprising. To note, data regarding the efficacy of frozen sperm samples are unclear and poorly explored in the literature. This issue deserves future specific investigations. Overall, the real rate of men with cancer who will achieve parenthood thanks to frozen semen is presumably below $5 \%$ (theoretically $2.6 \%$, calculated as $0.091 \times 0.63 \times 0.46$ ), corresponding to a number needed to be treated above 20 (theoretically 38 ).

Our study provides also some interesting findings on medical and biological variables influencing the usage rate. In particular, we reported a global rate of azoospermia after the end of treatments of $21 \%$, being only $4 \%$ for testicular cancer cases but raising to $32 \%$ $33 \%$ when considering men with non-testicular cancers (Table 2). Thus, on theoretical bases, only one in five patients (one in three for non-testicular cancers) could be expected to potentially benefit from sperm banking. In addition, one has to consider that a proportion of referred patients will not survive cancer (15\% in our case series; Table 4). Again, in our cohort, a substantial difference emerged for surviving according to cancer diagnosis, with rates of $2 \%, 21 \%$ and $41 \%$ for testicular, haematological and other cancers, respectively (Table 4). Overall, again on theoretical grounds, one could generally expect $18 \%$ of potential users $(0.21 \times 0.85)$. Interestingly, since the rates of azoospermia and survival pull in opposite directions for testicular cancer, the difference in the estimate of potential users is attenuated for men with testicular cancers $(0.04 \times 0.98=0.04$, thus $4 \%)$ compared to those with haematological cancers $(0.32 \times 0.79=0.25$, thus $25 \%)$ and other cancers $(0.33 \times 0.59=0.19$, thus $19 \%)$. This aspect should also be considered in future cost-beneficial analyses. Although we have not collected data on the type of cancer treatment performed, a variable to be taken into consideration in this area is the rate of azoospermia as an effect of specific oncological treatments as well as the pathology itself. With respect to this theme, even if it is outside the scope of this work, it is therefore possible that the more gonadotoxic treatments guide patients towards a greater use of ART.

Interestingly, these speculations are confirmed by the more formal statistical analyses performed in our study. Indeed, the Cox and logistic regression analyses revealed that age at the time of cryostorage, the type of cancer and the total number of frozen spermatozoa were significant predictors of the usage rate. Age was an expected finding (younger men may have not yet attempted to conceive while older men take advantage of the cryopreserved sample first) and, albeit statistically significant, the contribution of the total number of frozen spermatozoa seems marginal. The most interesting finding is the lower rate of use for men with a diagnosis of testicular cancer. To note, this is also in line with previous studies investigating the possible effect of cancer diagnosis on the rate of use. ${ }^{9,16}$ For the first time, we can here provide an explanation. As shown in Table 3, semen quality is lower at the time of cancer diagnosis for testicular cancer but the detrimental effects of cancer treatments are much less pronounced. The rate of azoospermia in particular is markedly lower, as also confirmed by the logistic regression analysis. These findings are in line with previous evidence. ${ }^{9}$ In understanding how many men return to use their frozen samples, a broad view of aspects of life, sexuality, gender identity, relationship status, women reproductive health, access to information and ART treatments can have a crucial role. However, it should be noted that most of these aspects cannot be investigated at the time of cryopreservation or can vary significantly between the diagnosis and the time of possible use of frozen sperm samples.

Even if information on the reasons for disposal of banked semen are reported in only half of cases, the available evidence also supports the higher chances of spermatogenesis resumption in men with testicular cancer (Table 4).

In this regard, it has to be recognized that even if it is intuitive to presume that the most plausible explanation for the low utilization rate of cryopreserved semen is the frequent resumption of spermatogenesis, only few studies have directly addressed the issue of natural fertility after cancer treatments. With the advent of ICSI, one should also consider that a consistent proportion of men with damaged but not compromised spermatogenesis can achieve pregnancy with ART using fresh semen. To note, the few available studies on the chances of natural or ART-mediated pregnancies were generally focused on specific malignancies, they did not weigh the possible benefits of ART with fresh semen and the rate of azoospermia was not definitely clarified. ${ }^{17-20}$ Another important question is the attitude of the patients towards frozen sperm disposal. In our population, the proportion of men who discarded semen is higher compared to what is reported in our recent review $(16 \%)^{5}$ but also in other recent contributions. ${ }^{6-8}$ We can speculate that different rules of sperm banking in different centres can influence this variable, with specific regards to costs of maintenance, availability of post-treatment sperm analysis and counselling. The active follow-up performed in the year preceding our analyses may have also contributed. It also should be highlighted that nearly half of patients asking for the elimination of sperm samples do not report specific reasons. On the contrary, a large proportion of patient decided to maintain cryopreservation for many years without having a precise plan of future use.

Some strengths and limitations of our study deserve to be commented. Regarding the formers, the most relevant are the large sample size, the long-term follow-up, the completeness of data due to the recent active survey and the availability of post-treatment semen analysis in a consistent proportion of patients. The first limitation of our study is missing detailed information on status of illness and comorbidities and lack of reliable information on the type of cancer treatments. To note, baseline characteristics of referred patients (mainly age and type of cancer) also changed over time (data not shown). In addition, one has to consider the confounding effects of other important factors such as improved survival rates, modifications of regimens of cancer treatment, increased incidence of malignancies in the reproductive age, shifting of the reproductive age, growing awareness among oncologists of the availability of sperm cryostorage for male fertility preservation, implementation of networks between fertility facilities and oncological units. ${ }^{21}$ Another limitation is that our aim was limited to the usage rate as the only 
outcome of fertility preservation; however, it is worth mentioning that usage rate is only one of the possible factors to be used for analysis of sperm banking beneficial effects and that this outcome does not consider other fundamental aspects of oncofertility that have emerged over the last decade encompassing quality of life in those diagnosed with cancer. Indeed, oncofertility care should be viewed as a multidisciplinary and comprehensive discipline that includes support and information about diagnosis and treatment of the specific cancer, discussion of cancer-related fertility issues and fertility preservation options, management of sexual or hormonal dysfunctions, contraception and psychosocial support. ${ }^{22}$ Similarly, we do not have in-depth information on many patients who, although not using the sample, decide to keep it cryopreserved for many years and on many patients who ask for disposal of their samples. Further studies should specifically consider the reasons behind the decisions to eliminate or keep the cryopreserved sample.

On the other hand, it must be considered that many of these aspects require a different type of analysis from our purposes. Furthermore, important information on the utilization rate can provide useful elements for the personalization of the model of care.

Lastly, as for the follow-up data on ART outcomes, we must recognize that our sample size (95 patients) is not very large and consequently the confidence intervals of the observed proportions are quite wide.

\section{5 | CONCLUSION}

The usage rate of frozen sperm in cancer patient is low, even extending the duration of follow-up. The ultimate proportion of men achieving parenthood with the use of banked samples can be estimated to be lower than $5 \%$. We believe that our results can be interesting as one of the useful elements for formal cost-beneficial analyses aimed at assessing the validity of fertility preservation programmes. They should take into consideration subgroups analyses according to cancer diagnosis (men with testicular cancers are those that presumably can benefit less) and evaluate possible interventions that could improve the economical value (such as prompt disposal of samples in case of spermatogenesis resumption) or alternative clinical approaches such as testicular sperm extraction or sperm donation programmes. Finally, these analyses should balance the costs not only with fatherhood but also with the potential beneficial psychological effects associated to sperm freezing and with ethical implications in the management of onclological diseases.

\section{DISCLOSURES}

The authors declare no competing interests.

\section{AUTHOR CONTRIBUTIONS}

SF, AP and ES designed the study; SF, CG, CD and SN acquired data; MR, AP managed and analysed data; SF, FF, ES and AP drafted the manuscript and interpreted all the data. All authors critically revised the paper for important intellectual content. All authors finally approved the paper in the present form. All authors agreed to be accountable for all aspects of the work in ensuring that questions related to the accuracy or integrity of any part of the work are appropriately investigated and resolved.

\section{DATA AVAILABILITY}

The datasets generated during and/or analysed during the current study are available from the corresponding author on reasonable request.

\section{ORCID}

Alessio Paffoni (iD https://orcid.org/0000-0003-3112-3161

\section{REFERENCES}

1. Tournaye H, Dohle GR, Barratt CL. Fertility preservation in men with cancer. Lancet. 2014;384:1295-1301.

2. A.S.R.M. Fertility preservation in patients undergoing gonadotoxic therapy or gonadectomy: a committee opinion. Fertil Steril. 2019;112:1022-1033.

3. Oktay K, Harvey BE, Partridge AH, et al. Fertility preservation in patients with cancer: ASCO clinical practice guideline update. J Clin Oncol. 2018;36:1994-2001.

4. National Institute for Health and Care Excellence. Fertility: Assessment and Treatment for People with Fertility Problems. London, UK: NICE, CG156; 2013.

5. Ferrari S, Paffoni A, Filippi F, Busnelli A, Vegetti W, Somigliana E. Sperm cryopreservation and reproductive outcome in male cancer patients: a systematic review. Reprod Biomed Online. 2016;33:29-38.

6. Depalo R, Falagario D, Masciandaro P, et al. Fertility preservation in males with cancer: 16-year monocentric experience of sperm banking and post-thaw reproductive outcomes. Ther Adv Med Oncol. 2016;8:412-420.

7. Shankara-Narayana N, Di Pierro I, Fennell C, et al. Sperm cryopreservation prior to gonadotoxic treatment: experience of a single academic centre over 4 decades. Hum Reprod. 2019;34:795-803.

8. Muller I, Ophuis RJAO, Broekmans FJM, Lock TMTW. Semen cryopreservation and usage rate for assisted reproductive technology in 898 men with cancer. Reprod Biomed Online. 2016;32(2):147-153. https://doi.org/10.1016/j.rbmo.2015.11.005

9. Ragni G, Somigliana E, Restelli L, Salvi R, Arnoldi M, Paffoni A. Sperm banking and rate of assisted reproduction treatment: insights from a 15-year cryopreservation program for male cancer patients. Cancer. 2003;97:1624-1629.

10. Ragni G, Arnoldi M, Somigliana E, Paffoni A, Brambilla ME, Restelli L. Reproductive prognosis in male patients with azoospermia at the time of cancer diagnosis. Fertil Steril. 2005;83:1674-1679.

11. Cooper TG, Noonan E, von Eckardstein S, et al. World health organization reference values for human semen characteristics. Hum Reprod Update. 2010;16:231-245.

12. Hosmer DWJ, Lemeshow S, Sturdivant RX. Wiley Series in Probability and Statistics. Applied logistic regression. 3rd ed. Hoboken, NJ: Wiley; 2013. https://onlinelibrary.wiley.com/doi/ book/10.1002/9781118548387.

13. Mills M, Rindfuss RR, McDonald P, te VE. Why do people postpone parenthood? Reasons and social policy incentives. Hum Reprod Update. 2011;17:848-860.

14. Eiser C, Arden-Close E, Morris K, Pacey AA. The legacy of sperm banking: how fertility monitoring and disposal of sperm are linked with views of cancer treatment. Hum Reprod. 2011;26:2791-2798. 
15. Pacey AA, Merrick H, Arden-Close E, et al. Monitoring fertility (semen analysis) by cancer survivors who banked sperm prior to cancer treatment. Hum Reprod. 2012;27:3132-3139.

16. Kelleher S, Wishart SM, Liu PY, et al. Long-term outcomes of elective human sperm cryostorage. Hum Reprod. 2001;16:2632-2639.

17. Menon S, Rives N, Mousset-Simeon N, et al. Fertility preservation in adolescent males: experience over 22 years at Rouen University Hospital. Hum Reprod. 2009;24:37-44.

18. Brydøy M, Fosså SD, Klepp O, et al. Paternity following treatment for testicular cancer. J Natl Cancer Inst. 2005;97(21):1580-1588. https://doi.org/10.1093/jnci/dji339

19. Ping $P, \mathrm{Gu} B H$, Li P, Huang YR, Li Z. Fertility outcome of patients with testicular tumor: before and after treatment. Asian J Androl. 2014;16:107-111.

20. Magelssen H, Haugen T, Vonduring V, Melve K, Sandstad B, Fossa S. Twenty years experience with semen cryopreservation in testicular cancer patients: who needs it? Eur Urol. 2005;48:779-785.
21. Hammarberg K, Kirkman M, Stern C, et al. Survey of reproductive experiences and outcomes of cancer survivors who stored reproductive material before treatment. Hum Reprod. 2018;33:179.

22. Anazodo A, Laws $P$, Logan $S$, et al. How can we improve oncofertility care for patients? A systematic scoping review of current international practice and models of care. Hum Reprod Update. 2018;25(2):159-179. https://doi.org/10.1093/humupd/dmy038

How to cite this article: Ferrari S, Paffoni A, Reschini M, et al. Variables affecting long-term usage rate of sperm samples cryopreserved for fertility preservation in cancer patients. Andrology. 2021;9:204-211. https://doi.org/10.1111/ andr.12894 